\title{
Quantum imaging with dielectric metasurfaces for multi-photon polarization tomography
}

\author{
Kai Wang ${ }^{1, *}$, Sergey S. Kruk ${ }^{1}$, Lei Xu ${ }^{1,2}$, Matthew Parry ${ }^{1}$, Hung-Pin Chung ${ }^{1,3}$, \\ Alexander S. Solntsev ${ }^{1}$, James Titchener ${ }^{1}$, Ivan Kravchenko ${ }^{4}$, Yen-Hung Chen ${ }^{3}$, \\ Yuri S. Kivshar ${ }^{1}$, Dragomir N. Neshev ${ }^{1}$, and Andrey A. Sukhorukov ${ }^{1}$ \\ ${ }^{1}$ Nonlinear Physics Centre, Research School of Physics and Engineering, \\ The Australian National University, Canberra, ACT 2601, Australia \\ ${ }^{2}$ The MOE Key Laboratory of Weak Light Nonlinear Photonics, School of Physics and TEDA Applied Physics \\ Institute, Nankai University, Tianjin 300457, China \\ ${ }^{3}$ Department of Optics and Photonics, National Central University, Jhongli 320, Taiwan \\ ${ }^{4}$ Center for Nanophase Materials Sciences, Oak Ridge National Laboratory, Oak Ridge, Tennessee 37831, USA
}

\begin{abstract}
We suggest and realize experimentally dielectric metasurfaces with high transmission efficiency for quantum multi-photon tomography, allowing for full reconstruction of pure or mixed quantum polarization states across a broad bandwidth.

OCIS codes: (350.4238) Nanophotonics and photonic crystals; (270.5585) Quantum information and processing; (160.3918) Metamaterials.
\end{abstract}

The control of quantum-polarization states of photons promises a variety of applications in quantum information technologies by employing their multi-particle nature and hence nontrivial effects such as entanglement. With fastgrowing imaging techniques, nowadays observation of single photons with fine resolution in both time- and spatial degrees of freedom is made possible, such as by means of an electron-multiplying CCD (EMCCD) [1]. However, measurement of quantum photon states conventionally relies on experimental approaches utilizing a series of reconfigurable optical elements in bulk setups [2].

We suggest and develop experimentally, for the first time to our knowledge, a new concept of quantum-polarization measurements with a single nanostructured metasurface. The operating principle is presented in Fig. 1(a): A metasurface spatially splits different components of photon polarization states, which then enables full reconstruction of the photon state based on the photon correlations with simple polarization-insensitive single-photon detectors or EMCCD cameras. The sub-wavelength thin structure provides an ultimate miniaturization, and can facilitate photon tomography by spatially-resolved imaging without a need for reconfiguration. Such parallel-detection approach promises not only better robustness and scalability, but also the possibility to study the dynamics of quantum states in real-time.

We design and fabricate an all-dielectric metasurface optimized for photon state reconstruction, which is achieved by separating specially chosen polarization projections [see Fig. 1(a)]. In contrast to the previously developed metasurfaces for classical polarization measurement [3,4], our design employs a generalized geometric-phase approach, which allows the choice of projection bases throughout the Poincare sphere. This is particularly important in realizing optimal-frame polarization state reconstruction with flexibility in choosing the number of projections, enabling robust measurement of the Stokes parameters in both classical and quantum regimes. The dielectric-material-based implementation of the metasurface [5,6] (see also the review article [7]) avoids plasmonic loss associated with metallic structures for transmitted light, which is especially important for quantum optical applications at low-photon levels.

We perform optical characterizations of the fabricated metasurface. We probe the metasurface with differently polarized laser beams (at a wavelength range of 1556-1650 nm) and capture the $k$-space images. The observed far-field patterns are in agreement with our simulations based on rigorous coupled wave analysis (RCWA) method [see examples for two polarizations in Fig. 1(b)], whereas additional central spots originating from fabrication imperfections only slightly lower the diffraction efficiency. Furthermore, we process the sets of images to fully characterize the polarization projective bases on a Poincaré sphere and find them to be in excellent agreement to our design [Fig. 1(c)], which are chosen to be in the optimal frame for the reconstruction of multi-photon polarization states. The metasurface has a very high average diffraction efficiency of $85 \%$, while both the efficiency and projective bases are sustained across a broad bandwidth of over $100 \mathrm{~nm}$. The condition number of the instrument matrix for single-photon state, i.e. the coefficient that the error is amplified in the inverse problem, is in the range of 2.0-2.7 for the measured band (1.73 for theoretically optimal frame). Therefore the fabricated metasurface can enable highly robust reconstruction of the polarization state. 


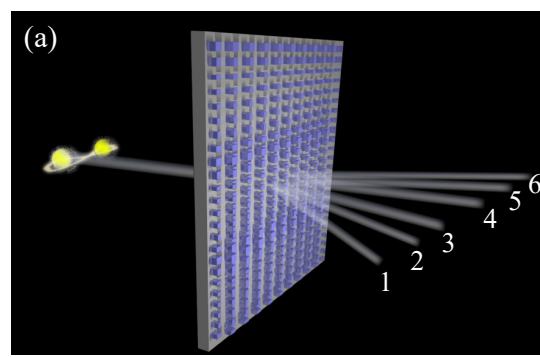

(b) Vertical linear polairzation Simulation

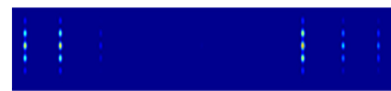

Experiment

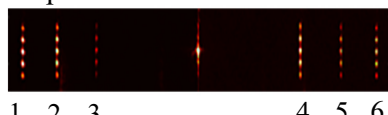

Left-hand circular polairzation Simulation

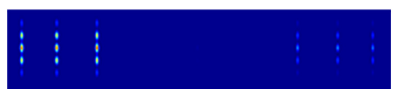

Experiment

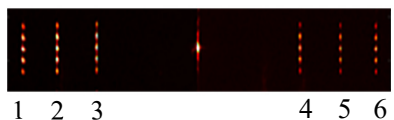

(c)
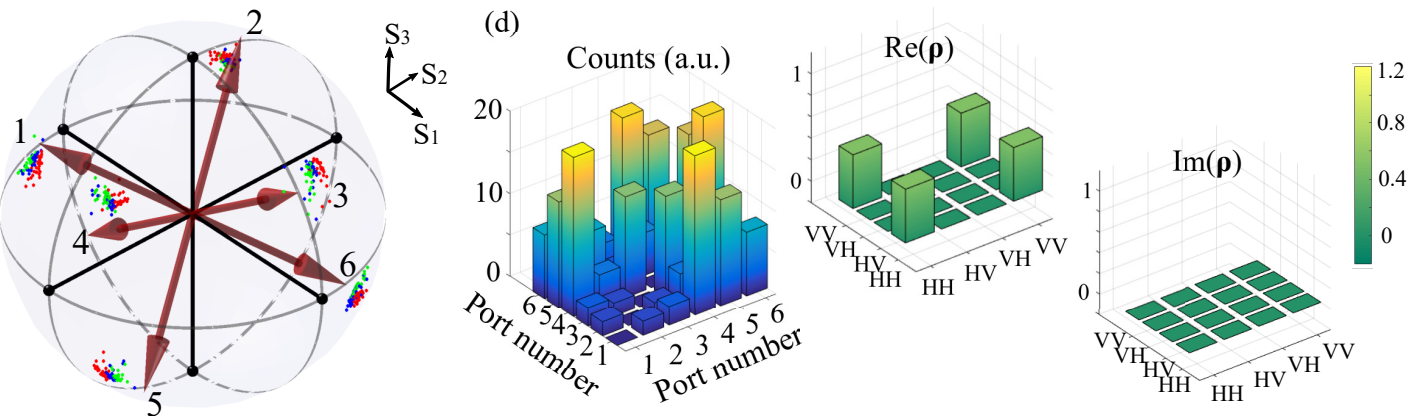

Fig. 1. (a) Concept: Dielectric metasurface for measurement of the quantum-polarization state of light, which separates a beam into several output ports (numbered 1 to 6) according to input polarization. (b) Experimentally measured and numerically simulated representative transmission patterns in $k$-space for different polarizations of an input Gaussian beam at $1570 \mathrm{~nm}$, as indicated by labels. (c) Measured (dots) and designed (arrows) polarization projective bases on a Poincaré sphere. The colors of dots denote wavelength ranges: 1556-1585 nm (red), 1586-1615 nm (blue), and 1616$1650 \mathrm{~nm}$ (green). (d) Simulated two-photon correlations for an input $|H H\rangle+|V V\rangle$ state (left) and the tomographically reconstructed density matrix $\rho$ (right) using experimental data from (c).

We then demonstrate that the metasurface is suitable for multi-photon state tomography. Using a set of projective bases which we measured experimentally for classical light, we simulate numerically the transmission of arbitrary polarization-entangled two-photon states through the metasurface, and calculate the two-photon correlations between the different outputs. We then show that the correlation measurements can be used to perform complete tomographic reconstruction of the input quantum polarization state of two photons, see an example in Fig. 1(d). Our metasurface with six output beams can measure up to four-photon polarization states using simple on-off single-photon detectors, whereas multi-photon states can be fully characterized by imaging on an EMCCD camera.

We anticipate that our approach will find applications in free-space quantum communication, cryptography, and computation, where metasurface can serve as a robust and accurate device for quantum imaging, replacing multiple bulk elements. Our metasurface may be extended to imaging spatially-varying quantum-polarization states, e.g. higher order multi-photon Stokes parameters.

The authors acknowledge financial support from the Australian Research Council. A portion of this research was conducted at the Center for Nanophase Materials Sciences, which is a DOE Office of Science User Facility.

\section{References}

1. M. P. Edgar, D. S. Tasca, F. Izdebski, R. E. Warburton, J. Leach, M. Agnew, G. S. Buller, R. W. Boyd, and M. J. Padgett, Nat. Commun. 3, 984 (2012).

2. D. F. V. James, P. G. Kwiat, W. J. Munro, and A. G. White, Phys. Rev. A 64, 52312 (2001).

3. A. Pors, M. G. Nielsen, and S. I. Bozhevolnyi, Optica 2, 716 (2015).

4. J. P. Balthasar Mueller, K. Leosson, and F. Capasso, Optica 3, 42 (2016).

5. A. Arbabi, Y. Horie, M. Bagheri, and A. Faraon, Nat. Nanotechnol. 10, 937 (2015).

6. S. Kruk, B. Hopkins, I. I. Kravchenko, A. Miroshnichenko, D. N. Neshev, and Y. S. Kivshar, APL Photonics 1, 30801 (2016).

7. A. I. Kuznetsov, A. E. Miroshnichenko, M. L. Brongersma, Y. S. Kivshar, and B. Lukyanchuk, Science 354, 2472 (2016). 